\title{
VEINTE AÑOS DE INVESTIGACIÓN Y DOCENCIA PUBLICITARIAS EN SEVILLA
}

Juan Rey (Universidad de Sevilla)

http://dx.doi.org/10.12795/AdMIRA.2009.01.14

Resumen: La actual Facultad de Comunicación de Sevilla comienza su andadura en octubre de 1989. En el presente trabajo se lleva a cabo un estudio de la trayectoria de los estudios de Publicidad en este periodo de tiempo. Para ello, primero, se cuenta la historia del Departamento de Comunicación Audiovisual y Publicidad, historia que, en los inicios, se confunde con la de la propia Facultad. Luego, se analiza la difícil y extraña posición que ocupa la Publicidad tanto en el contexto nacional como en el de la Facultad. Y por último se repasan los profesores que imparten publicidad, los trabajos de investigación y tesis doctorales sobre publicidad defendidos, los grupos de investigación que trabajan en publicidad, las jornadas y encuentros de publicidad celebrados, y las publicaciones dedicadas a la publicidad. Concluye el trabajo con unas perspectivas de los estudios de Publicidad.

Palabras clave: Publicidad, investigación, grupos de investigación, docencia, tesis doctorales.

\section{Introducción}

Se cumplen ahora veinte años del comienzo de los estudios universitarios de Publicidad $^{1}$ en Sevilla. El objetivo de este trabajo es dar cuenta, es decir, contar lo que ha sucedido en este tiempo y en estos estudios. Puede que para el tanguista veinte no sean nada, pero para los que han visto el nacimiento de estos estudios y han seguido de cerca su crecimiento, veinte años son un periodo considerable y, por qué no, entrañable. Un periodo lleno de dudas y titubeos, sueños y esperanzas, desvelos e inquietudes, afanes y aspiraciones. Un periodo pleno, en el que un grupo de profesores ha visto crecer y desarrollarse unos estudios en los que ha ido poniendo, poco a poco, parte de su propia vida. Cuanto se relata a continuación es fruto, en cierto modo, de la experiencia personal del autor que desembarcó en la nueva Facultad en sus primeros meses y aún sigue en ella, formando y orientando a sus colegas, muchos de los cuales se han independizado ya de su tutela paterno-académica y pueden relevarlo con creces en sus funciones burocrático-investigadoras, pues en estos años ellos, como discípulos aplicados y aprendices expertos, han ido creciendo y desarrollándose al compás y a la sombra de los estudios de Publicidad en la Facultad de Comunicación de Sevilla.

\section{Historia de una facultad y un departamento}

La historia del actual departamento de Comunicación Audiovisual y Publicidad está indisolublemente ligada a la de la Facultad con la que se entremezcla en sus primeros años. La actual Facultad de Comunicación de Sevilla comienza su trayectoria en octubre

\footnotetext{
${ }^{1}$ En este texto, el término "Publicidad" es polivalente. Unas veces, por simplicidad, se utiliza como sinónimo de la licenciatura en Publicidad y Relaciones Públicas y otra se emplea como equivalente de los estudios y/o actividades de comunicación (empresarial, comercial, institucional, política...).
} 
de 1989 en un edificio de la calle Gonzalo Bilbao, edificio que venía a ser una amalgama de varias casas agregadas y añadidas al chalé que el pintor sevillano tenía en las entonces afueras de la ciudad y que, con el tiempo, terminaron convertidas en la Escuela Superior de Bellas Artes y que, una vez transformada en Facultad y trasladada a la plaza de la Encarnación, deja un vasto inmueble que ha ido siendo ocupado temporalmente por centros de nueva creación hasta su ubicación definitiva: Psicología, Ciencias del Trabajo... Uno de estos centros transitorios es la nueva Facultad que recibe la pomposa denominación de Facultad de Ciencias de la Información, nombre que plantea algunos problemas, ya que, por una parte, pretende otorgar, como en otros muchos casos, el rango científico a unas actividades que, en cierto modo, están más próximas de la técnica, en el sentido griego del término, que de la ciencia, y, por otra, alude en exclusiva a la licenciatura de Periodismo dejando fuera a la de Comunicación Audiovisual y Publicidad. Este error fue subsanado varios años después cuando, en una Junta de Facultad, tras un ligero debate entre los partidarios de mantener el término Ciencia y los detractores, se le cambió finalmente el nombre por el más ajustado de Facultad de Comunicación.

Los primeros profesores de la nueva Facultad proceden de diversos departamentos de la Universidad de Sevilla y los que luego constituirán el departamento de Comunicación Audiovisual y Publicidad -en sus múltiples fases- están adscritos al Departamento de Literatura Española, que es el departamento matriz de la Facultad, ya que el primer Decano -Decano Comisario- es Jorge Urrutia, catedrático de Literatura Española y principal promotor, gestor y animador de la Facultad. Por estas fechas, las relaciones entre el departamento de Literatura Española y sus ahijados de Ciencias de la Información no son cordiales. Las desavenencias entre los docentes de ambos centros y los múltiples problemas que los nuevos profesores generan al departamento de Literatura Española con el que no se sienten identificados -no sólo los separa la concepción del mundo universitario sino también la ubicación: uno en la Puerta del Osario y otro en la calle San Fernando, a lo que debe sumársele el rápido crecimiento del nuevo centro- da lugar en 1991 al nacimiento del primer departamento ubicado en la Facultad y con una denominación más o menos específica: Departamento de Comunicación Audiovisual y Publicidad, Periodismo, Literatura y Estética. Si el edificio es un añadido de antiguas y ajenas viviendas, el nuevo departamento es, asimismo, una suma de diversas áreas de conocimiento.

Como se ha indicado, los primeros son años de confusión en los que no se sabe muy bien dónde termina la Facultad y dónde comienza el Departamento. Incluso el mismo Departamento es un mosaico de reinos de taifas en el que cada área de conocimiento gestiona su docencia al tiempo que no se inmiscuye en la de las restantes. Si a ello se le añade la continua y masiva contratación de profesores, muchos de ellos procedentes del mundo de la profesión, lo que genera un ambiente de novatos y advenedizos al ámbito universitario, se tendrá una idea aproximada de los comienzos caóticos del nuevo Departamento cuya historia inicial se confunde con la de la propia Facultad.

Poco a poco, la situación comienza a normalizarse burocráticamente. Tras diversos cursos académicos y varios decanos ${ }^{2}$ la Facultad sale adelante a pesar de las

\footnotetext{
2 El Decano Comisario, Jorge Urrutia, abandona el cargo y es nombrado José Manuel López Arenas. Las esperanzas en él depositadas se desvanecen enseguida pues fallece al poco tiempo. Tras un interregno de inseguridad e indecisión, pues nadie opta al cargo, tampoco hay Profesores Titulares ni docentes con
} 
adversidades. A ello contribuyen dos factores. Uno: lo que en cierto modo puede denominarse la simplificación del Departamento de Comunicación: Estética se segrega en 1994 y Periodismo, en 1998. Y dos, consecuencia del anterior: la consolidación del profesorado que paulatinamente va obteniendo titularidades, lo que supone la creación de un plantel de profesores capaces de asumir, en primer lugar, la docencia con garantías y, en segundo lugar, la formación de nuevos investigadores. El traslado al nuevo edificio de la Cartuja, prometido para 1993 y postergado hasta 2004, significa la consolidación de la Facultad de Comunicación, en cuyo flamante edificio se ubican los tres departamentos específicos (Comunicación Audiovisual y Publicidad, Periodismo I y Periodismo II), departamentos que, junto a otros muchos de la Universidad de Sevilla, con mayor o menor presencia docente, han contribuido a que la Facultad de Comunicación se haya hecho un hueco en el contexto universitario español. Y de todos estos departamentos, el de Comunicación Audiovisual y Publicidad es el más numeroso, el que mayor carga docente soporta y el de mayor responsabilidad al ser el encargado de gestionar dos grados: Comunicación Audiovisual y Publicidad y Relaciones Públicas.

\section{La difícil y extraña posición de la Publicidad}

En la actual organización del sistema universitario español, la publicidad no es un área de conocimiento. Ocupa una posición ambigua. Es un casillero polivalente. Por un lado, forma parte del nombre de una de las áreas de conocimiento reconocidas oficialmente: Comunicación Audiovisual y Publicidad. Pero, por otra, constituye la denominación de uno de los grados oficiales: Publicidad y Relaciones Públicas. Al no ser área de conocimiento, no puede constituir un departamento, a pesar de soportar un grado. No obstante estas dificultades, los profesores de Publicidad en Sevilla han logrado constituir un grupo que, además de por sus cordiales relaciones personales, se caracteriza por el rigor de su profesionalidad, la excelencia de sus investigaciones y la alta consideración de su docencia.

\subsection{Profesores}

A fecha de 30 de septiembre de 2009, el número de profesores que imparten disciplinas de Publicidad es de veinte que, sobre un total de cincuenta y cinco que conforman el Departamento de Comunicación Audiovisual y Publicidad, representa un 36,36 por ciento (véase la tabla 1).

\begin{tabular}{|l|l|}
\hline $\begin{array}{l}\text { Tabla 1. Profesores de CAP que } \\
\text { imparten Publicidad }\end{array}$ \\
\hline Catedrático de Universidad & 0 \\
\hline Titular de Universidad & 8 \\
\hline Contratado doctor & 2 \\
\hline Ayudante doctor & 2 \\
\hline
\end{tabular}

experiencia, el Rectorado nombra decano a José Manuel Gómez \& Méndez, el único Profesor Titular Interino. 


\begin{tabular}{|l|l|}
\hline Asociado & 6 \\
\hline Ayudante no doctor & 1 \\
\hline Becario FPI & 1 \\
\hline Total & $\mathbf{2 0}$ \\
\hline
\end{tabular}

Este porcentaje de profesores, bien formados, con relevantes currículos, dedicados a impartir en exclusiva disciplinas relacionadas con la Publicidad y con un notable reconocimiento nacional, podría constituir el embrión de lo que un día pudiera ser el Departamento de Comunicación Audiovisual y Publicidad II.

\subsection{Trabajos de investigación y tesis doctorales}

El alumnado que se matricula en la licenciatura de Publicidad y Relaciones Públicas lo hace con las miras puestas casi exclusivamente en la profesión. De ahí que, una vez concluidos el Primer y Segundo Ciclo, muy pocos continúen los estudios universitarios. Esta marcada opción laboral genera un problema colateral: la falta de profesores y, más que la falta de profesores, la falta de profesores cualificados, ya que muy pocos se inclinan por la docencia. Ciertamente, la carrera académica es lenta y enojosa, cuando no ingrata y desalentadora. A pesar de estas dificultades -la opción laboral de la mayoría y la desafección de la carrera universitaria-, siempre hay un alumno que solicita información sobre el Tercer Ciclo para continuar su formación universitaria en el ámbito de la Publicidad.

Si bien este problema afecta en general al alumnado de la Facultad de Comunicación, centro eminentemente práctico y profesional, incide con mayor virulencia en los matriculados en Publicidad y Relaciones Públicas. Según la tabla 2, de un total de ciento veinte trabajos de investigación leídos en el Departamento a lo largo de sus veinte años de existencia -en los que hay que considerar la constitución multitudinaria de los primeros años y las diversas segregaciones-, de este total de trabajos, sólo dieciséis versan sobre materias publicitarias y afines, lo que supone nada más que un 13 por ciento del total de trabajos defendidos, un porcentaje verdaderamente exiguo, pero cuya causa hay que buscarla, como se ha indicado, en la salida profesional por la que opta la mayoría del alumnado.

\begin{tabular}{|c|c|c|}
\hline \multicolumn{3}{|c|}{$\begin{array}{l}\text { Tabla 2. } \quad \begin{array}{c}\text { Trabajos } \\
\text { investigación defendidos }\end{array} \text { en el } \\
\text { Departamento }\end{array}$} \\
\hline Publicidad & 16 & $13 \%$ \\
\hline Com. Audiovisual & 51 & $43 \%$ \\
\hline Periodismo & 26 & $22 \%$ \\
\hline Literatura & 23 & $19 \%$ \\
\hline Estética & 4 & $3 \%$ \\
\hline Total & 120 & \\
\hline
\end{tabular}


Los dieciséis trabajos de investigación que en estos veinte años se han defendido abarcan los más diversos temas, siempre relacionados con la Publicidad. En la tabla 3 puede verse la relación de trabajos defendidos, con indicación del autor y la fecha.

Tabla 3. Relación de trabajos de investigación sobre PUB\&RRPP

\begin{tabular}{|c|c|c|}
\hline Título & autor & año \\
\hline $\begin{array}{l}\text { Semiótica y publicidad. Un caso práctico: los } \\
\text { anuncios de vino. }\end{array}$ & Juan Rey Fuentes & 1993 \\
\hline $\begin{array}{l}\text { El gabinete de Comunicación: Teoría y práctica } \\
\text { de una acción de Relaciones Públicas. }\end{array}$ & $\begin{array}{l}\text { Alejandro } \\
\text { Antona Illanes }\end{array}$ & 1993 \\
\hline $\begin{array}{l}\text { Iconología del cuerpo masculino en el discurso } \\
\text { publicitario de cosméticos. El caso de los } \\
\text { perfumes. }\end{array}$ & $\begin{array}{l}\text { Paloma Abad de } \\
\text { los Santos }\end{array}$ & 2001 \\
\hline $\begin{array}{l}\text { El sitio web integrado en la estrategia de } \\
\text { comunicación comercial, en el sector } \\
\text { alimenticio. }\end{array}$ & $\begin{array}{l}\text { María Gálmez } \\
\text { Cerezo }\end{array}$ & 2001 \\
\hline $\begin{array}{l}\text { El acto de posesión del Excmo. Sr. D. Manuel } \\
\text { Chaves como Presidente de la Junta de } \\
\text { Andalucía. }\end{array}$ & Marta Pulido Polo & 2001 \\
\hline $\begin{array}{l}\text { La imagen de Sevilla. Análisis corporativo } \\
\text { multidisciplinar sobre la imagen de Sevilla. }\end{array}$ & $\begin{array}{l}\text { Carmen Lasso de } \\
\text { la Vega }\end{array}$ & 2001 \\
\hline $\begin{array}{l}\text { La comunicación empresarial. Diagnóstico en la } \\
\text { promoción de Sevilla. }\end{array}$ & $\begin{array}{l}\text { Reyes León } \\
\text { Vergara }\end{array}$ & 2001 \\
\hline Modelos de propaganda. & $\begin{array}{l}\text { Antonio Pineda } \\
\text { Cachero }\end{array}$ & 2001 \\
\hline $\begin{array}{l}\text { El mensaje publicitario como configurador del } \\
\text { espacio social. Una aproximación teórica. }\end{array}$ & $\begin{array}{l}\text { Pedro A. Hellín } \\
\text { Ortuño }\end{array}$ & 2002 \\
\hline $\begin{array}{l}\text { El patrocinio empresarial a través de } \\
\text { fundaciones. La responsabilidad social en las } \\
\text { organizaciones. }\end{array}$ & $\begin{array}{l}\text { Auxiliadora } \\
\text { Alfaro Lara }\end{array}$ & 2004 \\
\hline $\begin{array}{l}\text { Análisis de la publicidad exterior de la campaña } \\
\text { Contradicciones de la marca Nobel. } \\
\text { Aproximación a una lectura simbólica de nuestro } \\
\text { ecosistema urbano. }\end{array}$ & $\begin{array}{l}\text { Dolores Cabral } \\
\text { Martín }\end{array}$ & 2004 \\
\hline $\begin{array}{l}\text { Conceptualización y contextualización del } \\
\text { videoclip como herramienta de comunicación } \\
\text { comercial del sector fonográfico. }\end{array}$ & David Selva Ruiz & 2006 \\
\hline $\begin{array}{l}\text { Análisis del arte como recurso expresivo del } \\
\text { discurso publicitario gráfico en España. }\end{array}$ & $\begin{array}{l}\text { Gloria Jiménez } \\
\text { Marín }\end{array}$ & 2006 \\
\hline $\begin{array}{l}\text { Violencia hacia la política y Opinión Pública. } \\
\text { Análisis comparativo entre España y Dinamarca. }\end{array}$ & $\begin{array}{l}\text { Verónica } \\
\text { Guardiola Corral }\end{array}$ & 2007 \\
\hline $\begin{array}{l}\text { Aproximación al estudio y contextualización del } \\
\text { tráiler cinematográfico. }\end{array}$ & $\begin{array}{l}\text { Javier } \\
\text { Delmar }\end{array}$ & 2009 \\
\hline Mito, comunicación y persuasión. & $\begin{array}{l}\text { María del Mar } \\
\text { Rubio Hernández }\end{array}$ & 2009 \\
\hline
\end{tabular}


En cuanto a las tesis doctorales, el fenómeno es similar (véase la tabla 4). De un total de setenta y una tesis defendidas, sólo trece corresponden a Publicidad. Aunque el porcentaje de tesis defendidas es del 18 por ciento, es decir, ha subido ligeramente respecto a los trabajos de investigación, sigue siendo inferior al de las defendidas en Comunicación Audiovisual. Cabe indicar que el bajo número de tesis de Periodismo se debe a la pronta segregación de dicha área de conocimiento y la consiguiente constitución de un departamento autónomo.

\begin{tabular}{|l|l|l|}
$\begin{array}{l}\text { Tabla 4. Tesis } \\
\text { defendidas en el Departamento } \\
\text { dectorales }\end{array}$ \\
\hline Publicidad & 13 & $18 \%$ \\
\hline Com. Audiovisual & 34 & $48 \%$ \\
\hline Periodismo & 16 & $23 \%$ \\
\hline Literatura & 7 & $10 \%$ \\
\hline Estética & 1 & $1 \%$ \\
\hline Total & 71 & \\
\hline
\end{tabular}

Las trece tesis doctorales que en estos veinte años se han realizado abarcan los más diversos temas, siempre relacionados con la Publicidad. En la tabla 5 puede verse la relación de tesis defendidas, con indicación del autor y la fecha.

tabla 5. Relación de tesis doctorales sobre PUB\&RRPP

\begin{tabular}{|l|l|l|}
\hline título & autor & año \\
\hline Los modelos masculinos en publicidad impresa. & Juan Rey Fuentes & 1994 \\
\hline $\begin{array}{l}\text { Los Medios de Comunicación en Andalucía: } \\
\text { características diferenciales de las audiencias. }\end{array}$ & $\begin{array}{l}\text { Carlos Guerrero } \\
\text { Serón }\end{array}$ & 1995 \\
\hline $\begin{array}{l}\text { Ordenación de la legislación publicitaria a partir } \\
\text { de la Ley de la Publicidad. }\end{array}$ & $\begin{array}{l}\text { Alfredo Sanz } \\
\text { Díaz }\end{array}$ & 1996 \\
\hline $\begin{array}{l}\text { Creación de opinión, creación de imagen y y } \\
\text { proyecto político: el nacimiento del Euro. }\end{array}$ & $\begin{array}{l}\text { Antonio Cascales } \\
\text { Ramos }\end{array}$ & 1999 \\
\hline $\begin{array}{l}\text { Protocolo y Relaciones Públicas de Estado. Los } \\
\text { días nacionales en la Exposición Universal de } \\
\text { Sevilla. }\end{array}$ & $\begin{array}{l}\text { María Teresa } \\
\text { Otero Alvarado }\end{array}$ & 2000 \\
\hline $\begin{array}{l}\text { La Guerra Civil y el Nuevo Estado. Una visión a } \\
\text { través de la publicidad en el diario ABC de } \\
\text { Sevilla (1936-1939). }\end{array}$ & $\begin{array}{l}\text { Juan } \\
\text { Rodríguez } \\
\text { Centeno }\end{array}$ & 2002 \\
\hline $\begin{array}{l}\text { La representación de la violencia en los spots } \\
\text { publicitarios emitidos por Canal Sur Televisión } \\
\text { (1999-2000). }\end{array}$ & $\begin{array}{l}\text { Manuel Garrido } \\
\text { Lora }\end{array}$ & 2002 \\
\hline
\end{tabular}




\begin{tabular}{|l|l|l|}
\hline $\begin{array}{l}\text { La humanización de la personalidad empresarial. } \\
\text { El estudio de la axiología corporativa transmitida } \\
\text { por los mensajes publicitarios. }\end{array}$ & $\begin{array}{l}\text { Rafael Vidal } \\
\text { Jiménez }\end{array}$ & 2003 \\
\hline $\begin{array}{l}\text { Imagen vs identidad. Análisis corporativo } \\
\text { multidisciplinar sobre la imagen comunicativa y } \\
\text { la identidad de Andalucía. }\end{array}$ & $\begin{array}{l}\text { Carmen Lasso de } \\
\text { la Vega }\end{array}$ & 2004 \\
\hline $\begin{array}{l}\text { Elementos para una teoría comunicacional de la } \\
\text { Propaganda. }\end{array}$ & $\begin{array}{l}\text { Antonio Pineda } \\
\text { Cachero }\end{array}$ & 2005 \\
\hline $\begin{array}{l}\text { Los gabinetes de protocolo como herramienta de } \\
\text { Relaciones Públicas en las Universidades } \\
\text { Españolas en el siglo XXI. }\end{array}$ & $\begin{array}{l}\text { Salvador } \\
\text { Hernández } \\
\text { Martínez }\end{array}$ & 2006 \\
\hline $\begin{array}{l}\text { Relaciones Públicas yesponsabilidad Social. } \\
\text { La fundación de empresa como estrategia de } \\
\text { reputación organizacional. }\end{array}$ & $\begin{array}{l}\text { Auxiliadora } \\
\text { Alfaro Lora }\end{array}$ & 2006 \\
\hline $\begin{array}{l}\text { El videoclip como herramienta de comunicación } \\
\text { comercial del sector fonográfico. }\end{array}$ & David Selva Ruiz & 2009 \\
\hline
\end{tabular}

\subsection{Grupos de investigación}

Puede afirmarse sin ambages que, en la Facultad de Comunicación de Sevilla, la investigación en Publicidad, en su sentido más amplio, se desarrolla al amparo del grupo de investigación en Métodos, análisis y estrategias de la comunicación empresarial e institucional (MAECEI), adscrito al Plan Andaluz de Investigación. Su director es el profesor Juan Rey. El grupo MAECEI es el primero que se crea en el departamento de Comunicación Audiovisual y Publicidad. Nace en 1992 y desde 2000 recibe ayudas a la investigación con regularidad. En las cuatro últimas convocatorias ha sido el grupo que ha recibido mayor subvención, lo que indica la sobresaliente actividad investigadora de sus integrantes. Su objetivo, como expresa su denominación, es el estudio de todas las cuestiones relacionadas con la comunicación empresarial e institucional. MAECEI es, pues, el promotor de la investigación publicitaria en Sevilla y ello puede observarse tanto en la cantidad de encuentros, jornadas y cursos que sobre Publicidad ha organizado (véase el apdo. 3.4) como en el número de publicaciones que ha promovido (véase el apdo. 3.5).

MAECEI es además el único grupo histórico del Departamento que está adscrito al área de las Ciencias Sociales (SEJ-197/1992). Los grupos restantes pertenecen a la de Humanidades y están vinculados al mundo de la Comunicación Audiovisual. Por orden cronológico, son: Historia de la imagen en movimiento y música audiovisiva (HUM406/1994), dirigido por el profesor Carlos Colón; Historia del cine español y sus relaciones con las artes con las otras artes (HUM-443/1995), dirigido por el profesor Rafael Utrera; e Historia, lenguaje y tecnología audiovisuales (HUM-656/1996), dirigido por el profesor Francisco Perales. No obstante, el último grupo de investigación surgido en el Departamento está centrado en el ámbito de la comunicación audiovisual y adscrito al área de las Ciencias Sociales: Análisis de medios, imágenes y relatos audiovisuales en su historia para el cambio social (SEJ-027/2009), dirigido por la profesora Virginia Guarinos. Infelizmente, no puede decirse lo mismo del ámbito de la Relaciones Públicas, en el que, al principio, la profesora María Teresa Otero promovió 
un grupo de investigación centrado en las relaciones públicas, el ceremonial y el protocolo que no llegó a configurarse oficialmente.

\subsection{Jornadas, encuentros y másteres}

Un de las principales actividades de los profesores de Publicidad ha sido la organización de eventos relacionados con los más diversos temas publicitarios. En este sentido, el grupo MAECEI ha organizado las I, II y II Jornadas de Publicidad (1992, 1993 y 1994); los I, II, III, IV y V Encuentros de Comunicación Empresarial e Institucional (1994, 1995, 1996, 1997 y 1998); las I, II, III y IV Jornadas de Consumo Publicidad y Cultura (2002, 2003, 2004 y 2005). De todos estos eventos obtiene el grupo MAECEI el soporte financiero para editar las diversas publicaciones que figuran en el siguiente apartado. Y también para organizar, en colaboración la École Nationale de Commerce et de Gestion de la Université Abdelmalek Essaâdi, el I Curso Internacional de Comunicación Empresarial e Institucional (Tánger, junio de 2005).

Aunque el grupo no figura expresamente como gestor, sus miembros son los principales responsables -bien como director, bien como coordinadores, bien como profesorestanto del Curso de Experto Universitario en Dirección de Comunicación Empresarial e Institucional (2003-04 y 2004-05) como de su sucesor: el Máster Universitario (Título Propio de la Universidad de Sevilla) en Dirección de Comunicación Empresarial e Institucional (2005-06, 2006-07, 2007-08 y 2008-09). El Curso de Experto y el Máster son los únicos de esta naturaleza que se imparten tanto en la Universidad de Sevilla como en la Comunidad Andaluza y, tras sus seis ediciones, se ha consolidado como un auténtico referente en el ámbito de la Dirección de Comunicación tanto en España como en los países vecinos y al otro lado del Atlántico a tenor de la procedencia de los alumnos que se han matriculado. Cada año se inscriben alumnos procedentes de Italia, Francia, Suiza, Portugal, Marruecos y, sobre todo, Venezuela, Colombia, México, Argentina y Brasil. En última instancia, el fallido grupo de Relaciones Públicas, Ceremonial y Protocolo, llevó a cabo tres ediciones de un Máster Universitario en Relaciones Públicas y Protocolo (2003-04, 2004-05 y 2005-06) y organizó tres congresos internacionales sobre la misma materia.

\subsection{Publicaciones}

Además de las numerosas publicaciones, ya en forma de artículo, ya en forma de libro o capítulo de libro, de cada uno de los profesores que imparten Publicidad, a la hora de analizar las publicaciones académicas sobre materias publicitarias, es inevitable referirse a la revista Questiones Publicitarias, la pionera del Departamento de Comunicación Audiovisual y Publicidad, pues aparece en 1993. Es indispensable destacar que es el grupo de investigación MAECEI, con sus diversas actividades, el editor, gestor y promotor de la revista.

Hasta la fecha se han editado catorce números. No obstante la historia de la revista no ha sido lineal, sino que ha sufrido diversos avatares económicos. En su trayectoria pueden distinguirse dos etapas, si bien la segunda consta de dos fases, según el soporte en el que aparezca. En la primera etapa, en la que simplemente se denomina Questiones 
Publicitarias, aparecen los primeros ocho números: 1 (1993), 2 (1994), 3 (1994), 4 (1995), 5 (1996), 6 (1997), 7 (1998) y 8 (1999). Tras un periodo de desaliento y desapoyo, surge con nuevos bríos y con un diseño más moderno. Pero no sólo cambia el aspecto, también se ajusta la revista a los requisitos exigidos por los catálogos científicos. En esta segunda etapa se llama Questiones Publicitarias. Revista Internacional de Comunicación y Publicidad. En ella puede distinguirse una primera fase durante la cual la revista aparece impresa. Son los números 9 (2004), 10 (2005) y 11 (2006). La segunda fase de la segunda etapa está constituida por los números 12 (2007), 13 (2008) y 14 (2009), todos ellos en versión telemática.

A partir del primer número de la segunda etapa ( $n^{\circ}$ 9, 2004), Questiones Publicitarias ingresa en la base de datos ISOC del CSIC y en LATINDEX (Sistema Regional de Información en Línea para Revistas Científicas de América Latina, el Caribe, España y Portugal). Asimismo pasa a formar parte del catálogo DICE (Difusión y Calidad Editorial de las Revistas Españolas de Humanidades y Ciencias Sociales y Jurídicas), patrocinado por el CSIC y CINDOC, y que es el referente que utiliza la ANECA para evaluar la calidad de las publicaciones. En última instancia, Questiones Publicitarias está incluida en IN-RECS, organismo que controla el índice de impacto de las revistas españolas de ciencias sociales y que está financiado por el Ministerio de Ciencia e Innovación.

Questiones Publicitarias no sólo ha sido la pionera del Departamento de Comunicación Audiovisual y Publicidad, también ha sido la primera y única revista de Publicidad en España. Durante muchos años ha sido el único referente editorial para los profesores de Publicidad. Es cierto que existen otras muchas revistas en el ámbito de la comunicación audiovisual, pero ninguna de ellas dedicada en exclusiva a la Publicidad como Questiones Publicitarias. Esto propició la aparición en sus páginas de interesantes y novedosos artículos sobre los más variados aspectos de la Publicidad. En 2007, aparece la excelente publicación Pensar la Publicidad. Revista Internacional de Investigaciones Publicitarias, editada conjuntamente por la Universidad Complutense de Madrid y la Universidad de Valladolid, lo que ha diversificado y ampliado el panorama de la publicación para los profesores de Publicidad.

Además de los números ordinarios, Questiones Publicitarias también ha publicado tres volúmenes monográficos. El primero es Algunas consideraciones sobre la comunicación empresarial e institucional (1997); el segundo, Comunicación política electoral. Elecciones municipales de Sevilla en 1999 (1999); y el tercero, Publicidad institucional (2009), que es el resultado de un proyecto $\mathrm{I}+\mathrm{D}$ llevado a cabo por los miembros del grupo MAECEI y profesores de la Universidad Pompeu Fabra de Barcelona.

Asimismo, MAECEI ha publicado dos volúmenes colectivos: Consumo, publicidad y cultura (2003) y Consumo y comunicación. Una aproximación plural (2004). Ambos son el resultado de unas Jornadas sobre Consumo, Publicidad y Cultura, organizadas por el grupo de investigación con la financiación de la Dirección General de Consumo de la Junta de Andalucía. Y ha propiciado la edición de otros dos: Los heraldos de acero. La propaganda de guerra y medios (2004) y Propaganda y comunicación. Una aproximación plural (2004), frutos ambos de las Jornadas de Propaganda. 
La última publicación auspiciada por el grupo MAECEI es Publicidad y sociedad. Un viaje de ida y vuelta (2008), volumen de casi trescientas páginas en el que, además de los miembros del grupo, participan profesores de diversas universidades españolas, lo que da una idea de las excelentes relaciones académicas de los profesores de Publicidad de la Universidad de Sevilla con otras universidades españolas.

No sería adecuado concluir este apartado sin hacer referencia a Laurea Hispalis. Revista Internacional de Relaciones Públicas, Ceremonial y Protocolo, revista auspiciada por el fallido grupo de investigación en Relaciones Públicas, Ceremonial y Protocolo que publicó tres números $(2001,2002$ y 2003).

\section{Perspectivas}

Después de veinte años de estudios de Publicidad en Sevilla, puede afirmarse que la situación tanto del profesorado de Publicidad como de la propia licenciatura (grado, según el protocolo de Bolonia) presenta un balance positivo. Veinte años han sido un largo camino plagado de obstáculos, sinsabores y desalientos. Pero también de alegrías, triunfos y logros. Ha habido de todo. Y los responsables, si hay algún responsable de todo ello, han sido los profesores que, con su esfuerzo, su tenacidad y su dedicación tanto investigadora y docente como administrativa y burocrática- han logrado no sólo consolidar los estudios de Publicidad, sino elevarlos de categoría.

Si se hace un recorrido por los diversos planes de estudio de Publicidad que a lo largo de estos dos decenios se han impartido en la Facultad de Comunicación de Sevilla, se observa que hay una progresión hacia la especialización. En esta trayectoria se distinguen tres etapas. Puede afirmarse, en términos generales, que el alumnado de las primeras promociones no cursaba Publicidad, sino que estudiaba la licenciatura de Periodismo con algunas asignaturas de Publicidad en los cursos superiores. Posteriormente, los alumnos de Publicidad cursaban un primer ciclo de Periodismo y un segundo de Publicidad. Y por último, el alumnado de Publicidad cursará un grado de Publicidad específico desde el primer año académico. Se trata, obviamente, de los planes de Bolonia, que están en fase de aprobación. Este ha sido el gran logro del profesorado de Publicidad: luchar por conseguir un grado específico en Publicidad, un grado acorde con las nuevas especializaciones, un grado capaz de formar al alumnado tanto para ejecutar eficazmente una profesión como para reflexionar sobre su función laboral y social.

La mejor radiografía de los estudios de Publicidad es aplicarle -someramente- un análisis DAFO.

Las fortalezas están constituidas por el profesorado, la demanda social y el nuevo plan de estudios. Como se ha indicado, el propio profesorado, con su juventud, su ímpetu y su afán de superación, ha logrado ubicar los estudios de Publicidad de Sevilla en el ámbito nacional, pues cada año, según las encuestas llevadas a cabo por el diario $E l$ Mundo, los estudios de Publicidad de Sevilla aparecen en tercer o cuarto lugar, posición que suele disputar con la Universitat Ramon Llull de Barcelona, ambas tras la Universidad Complutense de Madrid y la Universitat Autònoma de Barcelona. La gran demanda social que actualmente presentan los estudios de Publicidad es otra fortaleza. 
En Sevilla al menos ha superado a Periodismo y Comunicación Audiovisual. Lo cual demuestra el atractivo que para el alumnado presentan dichos estudios. En última instancia, el nuevo plan de estudio, según las directrices de Bolonia, es otra fortaleza, ya que su especialización ha de contribuir a atraer a aquellos alumnos que quieran formarse específicamente en Publicidad.

El profesorado, no obstante, constituye su principal debilidad. Más que el profesorado es exactamente la dificultad para reclutar profesores. Aunque en estos tiempos de crisis están comenzando a aparecer por el Departamento antiguos alumnos de Publicidad solicitando información tanto sobre los estudios de Tercer Ciclo como sobre la posibilidad de iniciar la carrera docente. Otra debilidad es el abandono en el que se encuentra la Facultad de Comunicación por parte de las instituciones. La no renovación periódica del material informático -ciertamente costoso y de vida efímera- incide negativamente en la formación del alumnado.

Las amenazas que sufren los estudios de Publicidad son la proliferación de centros dedicados a este tipo de estudios. Nacional, autonómica y localmente hay un crecimiento desmesurado de estos centros, en general carentes de profesorado formado y de respaldo legal. En cierto modo, este incremento de talleres, escuelas y academias "dedicados" a los estudios de Publicidad es consecuencia de la demanda social mencionada que, si bien se ha considerado una fortaleza, también representa una consecuencia indeseada. Otra amenaza es la saturación del mercado laboral. Andalucía dispone de tres centros universitarios dedicados a los estudios de Publicidad, que, por orden cronológico, son Sevilla, Málaga y Cádiz, sin embargo el tejido empresarial andaluz no tiene capacidad para asumir tantos profesionales de la Publicidad. Si a ellos se le suma que la alta demanda -es una licenciatura que está de moda, dicho coloquialmente- provoca que algunas facultades incrementen los grupos, como ha sucedido en Sevilla, puede suceder que la saturación del mercado laboral alcance cotas impredecibles.

Por último, las oportunidades pueden consistir en que el número de centros no oficiales dedicados a los estudios de Publicidad decaiga por la insuficiente formación que proporcionan a su alumnado, o que las empresas andaluzas se modernicen y comiencen a requerir los servicios de los profesionales de la Publicidad, o que aumente el número de medios locales y sean necesarios agentes publicitarios, o que finalice la crisis económica que experimenta Occidente, o que...

Esta ha sido la trayectoria de unos estudios que se han ido adecuando a los nuevos tiempos, a las nuevas necesidades sociales y a las nuevas tareas profesionales. Han sido veinte años de esfuerzos y logros en los que el profesorado de Publicidad ha tenido una gran responsabilidad. Sólo cabe desear que dentro de otros veinte años alguien escriba sobre los estudios de Publicidad en Sevilla e informe de que se ha superado con creces cuanto aquí se ha dicho. Que así sea.

\section{Bibliografía}


REIG, Ramón, y GARCÍA ORTA, María José (2000): “Investigación y comunicación en Sevilla. Once años de la Facultad de Ciencias de la Información (19892000)", en Ámbitos, no 5, pp. 259-311.

MAECEI, G.I. Archivo Particular.

MAECEI, G.I. Libro de Actas.

SIERRA CABALLERO, Francisco, y RAMÍREZ ALVARADO, María del Mar (2007): Catálogo de grupos de investigación, Sevilla, Facultad de Comunicación.

\section{Webgrafía}

$\underline{\text { www.dialnet.es }}$

www.fcom.us.es

www.investigación.us.es/sisius

$\underline{\text { www.maecei.es }}$

www.masterdcei.es

www.questiones.es 